\title{
Aplicación de método molecular en la detección de Mycoplasma genitalium en hombres y en mujeres embarazadas
}

\author{
Walter Gübelin H., M. Angélica Martínez T., Pablo Céspedes P., Félix Fich S., \\ H Fuenzalida C., Rodrigo de la Parra C., Luis Valderrama K., Solange Zapata M.
}

\section{Molecular detection of Mycoplasma genitalium in men and pregnant women}

Mycoplasma genitalium is an opportunistic pathogen of the genital tract. It has been implicated as an etiological agent of urethritis in men and cervicitis and pelvic inflammatory disease (PID) in women. The aim of this study was to determine the prevalence of Mycoplasma genitalium in male urethritis and in vaginal specimens of pregnant women. Urethral specimens obtained from 37 men presenting with urethritis and vaginal specimens from 50 consecutive pregnant women were tested for the presence of $M$. genitalium by polymerase chain reaction (PCR). The urethral specimens were also examined for the presence of Neisseria gonorrhoeae, Chlamydia trachomatis and Ureaplasma sp whereas the vaginal microbiota and the presence of genital mycoplasma were investigated in the vaginal specimens. Twenty three cases were classified as nongonococcal urethritis (NGU) and 14 as gonorrheal disease. M. genitalium was detected in 3 of 23 (13.04\%) men with NGU; in two cases with Ureaplasma sp, and in one patient as the unique agent. C. trachomatis was found in 7 patients with NGU and in one patient with gonorrhea. Ureaplasma sp was isolated in $13(35.1 \%)$ patients, 8 cases of NGU and in 5 patients with gonorrhea. The organism was also detected in $6(15 \%)$ of 40 women; in 5 cases in the presence of a normal microbiota (Nugent score 0-3), and an in one case in the presence of bacterial vaginosis. Ureaplasma spp was isolated in the 6 positive specimens. This study indicates that $M$. genitalium can be detected in urethral specimens of some cases of NGU as well as in the lower genital tract of pregnant women in the presence of a normal vaginal microbiota.

Key words: Mycoplasma genitalium, urethritis.

Palabras clave: Mycoplasma genitalium, uretritis.

\section{Introducción}

M ycoplasma genitalium fue aislado por primera vez en 1980 por Tully y cols en 2 de 13 hombres con síndrome de uretritis no gonocóccica $(\mathrm{UNG})^{1}$. No obstante, pese a que ya han transcurrido 25 años de su descubrimiento, su rol patológico está lejos de ser bien comprendido. El diagnóstico microbiológico de M. genitalium es difícil, porque crece lentamente en medios de cultivo, siendo rápidamente superado por Mycoplasma hominis y Ureaplasma sp, especies de frecuente aislamiento en el tracto genital ${ }^{2}$. Por este motivo su diagnóstico se basa en técnicas moleculares, como la hibridación con sondas y la amplificación del $\mathrm{ADN}$ mediante reacción de polimerasa en cadena (RPC) $)^{3-5}$. Varios estudios efectuados mediante RPC han detectado M. genitalium en 15 a $25 \%$ de hombres con $\mathrm{UNG}^{5-9}$. En la mujer, $M$. genitalium ha sido asociado con cervicitis ${ }^{5,10-11}$, enfermedad inflama- toria pelviana (EIP $)^{12-13}$ e infertilidad ${ }^{14}$. Sin embargo, como ocurre con los otros micoplasma genitales, el microorganismo también ha sido detectado en personas sin signos o síntomas de infección genital ${ }^{3,6}$. El objetivo de este estudio fue determinar la frecuencia de M. genitalium en hombres con uretritis y en muestras vaginales de mujeres sexualmente activas.

\section{Material y Métodos}

Pacientes y muestras clínicas. Período de estudio: $1^{\circ}$ de abril a 30 de noviembre de 2001. Varones con uretritis: se tomaron dos muestras de secreción uretral, en 37 hombres con diagnóstico clínico de uretritis, atendidos en los Servicios de Dermatología de los siguientes hospitales: Hospital Clínico de la Universidad de Chile, Hospital Clínico San Borja Arriarán, Hospital Barros Luco-Trudeau y Hospital San José. Las
Hospital Clínico Universidad de Chile

Departamento de Dermatología, (WGH, R de la PC, SZM)

Universidad de los Andes (WGH) Universidad de Chile

Facultad de Medicina, Instituto de Ciencias Biomédicas, Programa de Microbiología y Micología, (MAMT) Hospital San Juan de Dios Servicio de Obstetricia y Ginecología (PCP)

Hospital San Borja Arriarán Servicio de Dermatología, (FFS) Hospital Barros Luco-Trudeau Servicio de Dermatología, (HFC) Hospital San José

Servicio de Dermatología, (LVK)

Recibido: 16-05-05 Aceptado: 07-12-05

Correspondencia a: Walter Gübelin Harcha wgubelin@ctcinternet.cl 
muestras clínicas fueron obtenidas con tórula fina de algodón e inmediatamente inoculadas en medio de transporte Stuart y en 1,5 $\mathrm{ml}$ de buffer de transporte sacarosa fosfato (buffer 2SP). Mujeres sexualmente activas: se obtuvieron dos muestras de secreción vaginal de 40 mujeres atendidas consecutivamente en el Departamento de Alto Riesgo Obstétrico (DARO) del Hospital San Juan de Dios. En cada paciente se tomaron dos muestras de las paredes vaginales con tórulas de algodón.

Con la primera muestra se efectuó un frotis para tinción de Gram, depositándose luego la tórula en buffer 2SP destinado al cultivo de micoplasmas genitales y RPC. Las muestras en buffer 2SP fueron conservadas en refrigeración hasta su transporte al laboratorio e inmediatamente procesadas o congeladas a-70 ${ }^{\circ} \mathrm{C}$. La segunda muestra fue depositada en medio de transporte Stuart y destinada al cultivo de bacterias anaerobias facultativas y levaduras.

Microbiología. Reacción de polimerasa en cadena. Para el diagnóstico de M. genitalium mediante RPC se amplificaron $281 \mathrm{pb}$ del gen de la adhesina $\mathrm{MgPa}$ empleando los partidores descritos por Jensen y cols ${ }^{4}$. Para la extracción del ADN se centrifugó una alícuota de $0,4 \mathrm{ml}$ de la muestra clínica, a 13.000 r.p.m. durante $30 \mathrm{~min}$. El pellet resultante fue suspendido en $100 \mathrm{ml}$ de buffer de lisis $(10 \mathrm{mM}$ Tris- $\mathrm{HCl}, 50 \mathrm{mM} \mathrm{KCl}$, 2,0 $\mathrm{mM} \mathrm{MgCl}_{2}, 0,1 \%$ Tritón X-100 y $200 \mathrm{mg}$ por $\mathrm{ml} \mathrm{de}$ proteinasa $\mathrm{K}$ ). La mezcla fue incubada a $60{ }^{\circ} \mathrm{C}$ durante 60 min y luego a $94{ }^{\circ} \mathrm{C}$ durante 15 min y rápidamente enfriada. El ADN fue purificado por extracciones sucesivas con fenol cloroformo (fenol-cloroformo-alcohol isoamílico, 25: 24: 1) y cloroformo (cloroformo-alcohol isoamílico, 24: 1). Las reacciones de amplificación fueron efectuadas en un volumen de $50 \mathrm{ml}$ conteniendo: buffer de amplificación $1 \mathrm{X}, 2,5 \mathrm{U}$ Taq polimerasa $\left(\mathrm{Gibco}^{\mathrm{MR}}\right), 200 \mathrm{mM}$ de cada nucleótido trifosfato, 2,5 $\mathrm{mM} \mathrm{MgCl}_{2}$ y $1 \mathrm{mM}$ de cada partidor. Los ciclos de amplificación consistieron en 4 min de denaturación a $94^{\circ} \mathrm{C}$, seguidos por 40 ciclos de 1 min de denaturación a $94{ }^{\circ} \mathrm{C}, 1 \mathrm{~min}$ de anillamiento a $65{ }^{\circ} \mathrm{C}$ y $1 \mathrm{~min}$ de extensión a $72{ }^{\circ} \mathrm{C}$, con un ciclo final de extensión de 6 min a $72{ }^{\circ} \mathrm{C}$. Las amplificaciones fueron efectuadas en un termociclador MJ Research, Modelo MiniCycler ${ }^{\circledR}$. Los productos de amplificación fueron separados en función de su tamaño, por electroforesis en geles de agarosa al $1,5 \%$ conteniendo $0,5 \mathrm{mg}$ por $\mathrm{ml}$ de bromuro de etidio y posteriormente visualizados en transiluminador de luz ultravioleta. Como patrón de comparación del tamaño del ADN se utilizó ADN ladder de 100 pb. Como control positivo del proceso de amplificación se incluyó ADN de $M$ genitalium, cepa $\mathrm{G}-37^{\mathrm{T}}$, donado gentilmente por el Prof. Jorgen Skov Jensen, Copenhagen Dinamarca. Como control negativo se utilizó la mezcla de la reacción de RPC sin ADN.

Chlamydia trachomatis fue identificada mediante la amplificación de 517 pb del plasmidio críptico con partidores descritos por Claas y cols ${ }^{15}$. La RPC fue efectuada a partir del ADN extraído para la RPC de $M$. genitalium, por un procedimiento previamente descrito ${ }^{16}$.

Cultivos Para el diagnóstico de Ureaplasma sp se inoculó $0,1 \mathrm{ml}$ de buffer $2 \mathrm{SP}$ en dos tubos conteniendo $2 \mathrm{ml}$ de caldo urea, de acuerdo a procedimientos descritos anteriormente ${ }^{17-18}$. Brevemente, los caldos fueron incubados a $36{ }^{\circ} \mathrm{C}$ en aerobiosis y observados diariamente, durante $96 \mathrm{~h}$ para evidenciar el crecimiento de este microorganismo, dado por el cambio de $\mathrm{pH}$ hacia la alcalinización del medio. Para el diagnóstico de Neisseria gonorrhoeae la muestra en medio de transporte Stuart fue inoculada en agar Thayer Martin, luego de efectuarse un extendido para tinción de Gram. La identificación de $N$. gonorrhoeae fue efectuada por el examen directo teñido con Gram y confirmado por el aislamiento de diplococos gramnegativos oxidasa positiva en agar Thayer-Martin. El diagnóstico de síndrome de UNG fue efectuado en presencia de piocitos y ausencia de diplococos gramnegativos al examen directo de la muestra uretral teñida con Gram y un cultivo negativo en agar Thayer-Martin.

La microbiota vaginal fue clasificada en tres categorías: normal, intermedia y vaginosis bacteriana (VB), de acuerdo a la presencia y recuento microbiano en la tinción de Gram del frotis vaginal de los tres morfotipos vaginales más frecuentes: morfotipo lactobacilar, morfotipo cocobacilar y morfotipo de Mobiluncus sp. Se consideró microbiota normal a puntajes de 0-3 en la clasificación de Nugent, microbiota intermedia a recuentos 4-6 de la puntuación de Nugent y VB a frotis con 7-10 puntos $^{19}$.

\section{Resultados}

De los 37 pacientes con uretritis estudiados, 23 correspondieron a UNG y 14 a uretritis gonocóccica. Mycoplasma genitalium fue detectado en 3 de 23 $(13,04 \%)$ pacientes con UNG; en dos casos conjuntamente con Ureaplasma sp y en un caso como único agente (Tabla 1). Chlamydia trachomatis fue diagnosticada en $8(21,6 \%)$ pacientes; en 7 casos de UNG y en un paciente conjuntamente con $N$. gonorrhoeae. Se aisló Ureaplasma sp en $13(35,1 \%)$ pacientes; correspondiendo 8 casos a UNG y 5 casos a gonorrea. (Tabla 1). 
De las 40 muestras de secreción vaginal estudiadas, $29(72,5 \%)$ correspondieron a microbiota normal, 7 $(17,5 \%)$ a VB, $2(5 \%)$ a microbiota intermedia y en 2 muestras la calidad de la lámina no permitió la clasificación de la microbiota. Mycoplasma genitalium fue detectado en 6 de 40 (15\%) muestras vaginales (Tabla 2). En 5 casos la microbiota fue normal y en un caso correspondió a VB. En las 6 muestras positivas para $M$. genitalium también se aisló Ureaplasma sp. En la Tabla 2 se aprecia la frecuencia de detección de micoplasmas genitales en las muestras estudiadas.

\section{Discusión}

Chlamydia trachomatis constituye la principal causa del síndrome de UNG, aunque su incidencia en los países desarrollados ha disminuido en el tiempo por las medidas de control epidemiológico que se han instaurado $^{20}$. El 15 a 25\% de los casos agudos de UNG serían causados por M. genitalium y un porcentaje no bien determinado por Ureaplasma $\mathrm{sp}^{5-9,21}$.

Entre 20 y $60 \%$ de los pacientes con UNG aguda, aún tratados con una terapia antimicrobiana apropiada, desarrollan una uretritis crónica, siendo $M$. genitalium y Ureaplasma sp los agentes más frecuentemente detectados ${ }^{21}$. Hooton y cols investigaron mediante hibridación con sondas de ADN la presencia de M. genitalium en 203 hombres con diferentes tipos de uretritis, determinando la prevalencia del microorganismo según las preferencias sexuales de los pacientes. Se observó una asociación significativamente mayor de $M$. genitalium en pacientes homosexuales o bisexuales; 11 de 37 (30\%) versus 19 de 166 (11\%) hombres heterosexuales $(\mathrm{p}=0,009)$, sugiriendo que el tracto intestinal sería el reservorio de este microorganismo $^{3}$. Como ocurre frecuentemente con otros micoplasmas genitales, los autores detectaron también $M$. genitalium en 6 de 65 (9\%) y 4 de 19 (21\%) hombres heterosexuales y homosexuales, respectivamente, sin signos ni síntomas de infección genital (3). Infección uretral asintomática ha sido también descrita por Horner y cols, quienes identificaron $M$. genitalium en 24 de $103(23 \%)$ hombres con UNG y 3 de $53(6 \%)$ hombres sin uretritis $(\mathrm{p}<0,006)^{6}$.

Mycoplasma genitalium ha sido detectado en pacientes con cervicitis en algunos estudios ${ }^{4-5,10-11}$. Jensen y cols investigaron $M$. genitalium mediante RPC en 150 muestras cervicales y uretrales enviadas para búsqueda de $C$. trachomatis, detectando el microorganismo en $6,7 \%$ de muestras de mujeres y $12 \%$ de muestras de hombres ${ }^{4}$. Blanchard y cols investigaron $M$. genitalium mediante RPC en 114 pacientes con cervicitis o uretritis detectando el microorganismo en $14 \%$ de hombres y $6 \%$ de mujeres 5 . Uno y cols detectaron a $M$. genitalium en 5 de $64(7,8 \%)$ mujeres con cervicitis. Luego de la exclusión de las pacientes positivas para $C$. trachomatis, $M$. genitalium estuvo asociado con 5 de $57(8,8 \%)$ casos $^{10}$. Finalmente, Manhart y cols detectaron $M$. genitalium en 50 de 719 (7,0\%) muestras cervicales de pacientes con cervicitis mucopurulenta atendidas en una clínica de Enfermedades de Transmisión Sexual (ETS) ${ }^{11}$. Mycoplasma genitalium puede dar origen a una infección vaginal

\begin{tabular}{|c|c|}
\hline & \multirow{2}{*}{$\begin{array}{c}\text { Pacientes } \\
(n=37) \\
n \quad(\%)\end{array}$} \\
\hline & \\
\hline Neisseria gonorrhoeae & $14(37,8)$ \\
\hline Ureaplasma sp & $13(31,1)$ \\
\hline Chlamydia trachomatis & $8(21,6)$ \\
\hline Mycoplasma genitalium & $6(16,2)$ \\
\hline
\end{tabular}

\begin{tabular}{|c|c|c|}
\hline \multicolumn{3}{|c|}{$\begin{array}{c}\text { Tabla 2. Frecuencia de micoplasmas } \\
\text { genitales en secreción vaginal en } \\
40 \text { mujeres embarazadas } \\
\text { asintomáticas }\end{array}$} \\
\hline Especie & No & (\%) \\
\hline Ureaplasma sp & 32 & (80) \\
\hline Mycoplasma genitalium & 6 & (15) \\
\hline Mycoplasma hominis & 4 & (10) \\
\hline
\end{tabular}

ascendente, habiendo sido relacionado con EIP, tanto por la detección de su ADN por RPC, como mediante serología ${ }^{10,12-13}$. Los resultados de nuestro estudio aportan más interrogantes al significado de $M$. genitalium en el tracto genital femenino, ya que fue detectado en 5 pacientes con microbiota normal y sólo en una paciente con VB. Las muestras clínicas fueron obtenidas consecutivamente sin efectuar ninguna selección y la población estudiada se caracterizó por su baja frecuencia de VB (17,5\% de las pacientes). La prevalencia de VB en poblaciones atendidas en pacientes en Consultas de ETS suele llegar a $40 \%{ }^{22}$. En el estudio de Manhart y cols, M. genitalium fue asociado significativamente a edad joven y múltiples parejas sexuales en el último tiempo y negativamente con VB. Es posible que $M$. genitalium tenga un comportamiento similar al de Ureaplasma sp, microorganismo de transmisión sexual cuya presencia en la vagina sólo refleja actividad sexual. El reservorio de $M$. genitalium podría ser el ecosistema vaginal, pudiendo por extensión causar cervicitis e infecciones del tracto reproductivo superior. Casin y cols demostraron una alta prevalencia de $M$. genitalium en el tracto genital inferior de mujeres con flujo vaginal atendidas en una clínica de ETS, siendo detectado en forma significativamente 
mayor en la vagina (39\%) que en el cérvix (21\%) de las pacientes $^{23}$.

Mycoplasma genitalium posee la mínima cantidad de genes que se cree necesarios para permitir la vida en un organismo. Su genoma es de sólo $580 \mathrm{~kb}, 7,9$ veces más pequeño que el de Escherichia coli, lo que explica la naturaleza fastidiosa de este microorganismo. Tiene un organelo de adherencia y movilidad, "tip", similar al de la especie patógena Mycoplasma pneumoniae, con la cual comparte, además, una gran homología del $\mathrm{ADN}^{24}$. La asociación de $M$. genitalium con uretritis, cervicitis y EIP, señalan el potencial patogénico de este microorganismo.

En conclusión, nuestros resultados sugieren que M. genitalium es responsable de algunos casos de UNG, como único agente o asociado a otros patógenos genitales, y que puede formar parte de la microbiota vaginal comensal en algunas mujeres.

\section{Resumen}

Mycoplasma genitalium es un patógeno oportunista del tracto genital. En el hombre es causa de uretritis, en tanto que en mujeres ha sido implicado en la etiología de cervicitis y de enfermedad inflamatoria pelviana (EIP). El objetivo de este estudio fue determinar la prevalencia de $M$. genitalium en pacientes mas- culinos con uretritis y en muestras vaginales de mujeres embarazadas. Se obtuvo muestras de secreción uretral en 37 pacientes con uretritis y de muestras vaginales de 50 consecutivas mujeres embarazadas, determinándose la presencia de $M$. genitalium mediante reacción de polimerasa en cadena (RPC). Las muestras de secreción uretral fueron también evaluadas en busca de Chlamydia trachomatis, Neisseria gonorrhoeae y Ureaplasma sp en tanto que en las de origen vaginal se investigó la microbiota y presencia de micoplasmas de tipo genital. Veintitrés casos fueron clasificados como uretritis no gonocóccica (UNG) y 14 como enfermedad gonocóccica. M. genitalium fue detectado en 3 de 23 (13,04\%) varones con UNG; en dos casos asociado a Ureaplasma sp, y en un paciente como agente único. $C$. trachomatis fue detectado en 7 pacientes con UNG y en uno con gonorrea. Ureaplasma sp fue aislado en $13(35,1 \%)$ pacientes, 8 casos de UNG y en 5 con gonorrea. El microorganismo fue detectado también en 6 (15\%) de 40 mujeres; en 5 casos en presencia de microbiota normal (score de Nugent 0-3), y en un caso en presencia de vaginosis bacteriana. Ureaplasma spp fue aislado en las seis muestras positivas. En conclusión, este estudio demuestra que $M$. genitalium debe ser también considerado en la etiología de la UNG así como en el tracto genital inferior en la mujer embarazada, en presencia de una microbiota vaginal normal.

\section{Referencias}

1.- Tully J G, Taylor-Robinson D, Cole R M, Rose D L. A newly discovered mycoplasma in the human urogenital tract. Lancet 1981; 1: 1288-91.

2.- Jensen J S, Hansen H T, Lind K. Isolation of Mycoplasma genitalium strains from the male urethra. J Clin Microbiol 1996; 34: 286-91.

3.- Hooton T M, Roberts P L, Stamm W E, Roberts M C, Holmes K K, Kenny G E. Prevalence of Mycoplasma genitalium determined by DNA probe in men with urethritis. Lancet 1988; 1: 266-8.

4.- Jensen J S, Uldum S A, Sondergard-Andersen J, Vuust J, Lind K. Polymerase chain reaction for detection of Mycoplasma genitalium in clinical samples. J Clin Microbiol 1991; 29: 46-50.

5.- Blanchard A, Hamrick W, Duffy L, Baldus K, CasselL G H. Use of the polymerase chain reaction for detection of Mycoplasma fermentans and Mycoplasma genitalium in the urogenital tract and amniotic fluid. Clin Infect Dis 1993; 17 (Suppl I): S272-S279.

6.- Horner P J, Gilroy C B, Thomas B J, Naidoo R O, Taylor-Robinson D. Association of Mycoplasma genitalium with acute non-gonococcal urethritis. Lancet 1993; 342: 582-5.

7.- Maeda S, Tamaki M, Nakano M, Uno M, Deguchi T, Kawada Y. Detection of Mycoplasma genitalium in patients with urethritis. J Urology 1998; 159: 405-7.

8.- Totten P A, Schwartz M A Sjostrom K E, Kenny G E, Handsfield H H, Weiss J B, et al. Association of Mycoplasma genitalium with nongonococcal urethritis in heterosexual men. J Infect Dis 2001; 183: 269-76.

9.- Yoshida T, Deguchi T, Ito M, Maeda S, Tamaki M, Ishiko H. Quantitative detection of Mycoplasma genitalium from first-pass urine of men with urethritis and asymptomatic men by real-time PCR. J Clin Microbiol 2002; 40: 1451-5.

10.- Uno M, Deguchi T, Komeda H, Hayasaki M, Iida M, Nagatani M, et al. Mycoplasma genitalium in the cervices of Japanese women. Sex Transm Dis 1997; 24: 284-8.

11.- Manhart L E, Critchlow C W, Holmes K K, Dutro S M, Eschenbach D A, Stevens C E, et al. Mucupurulent cervicitis and Mycoplasma genitalium. J Infect Dis 2003; 187: 650-7.

12.- Moller B R, Taylor-Robinson D, Furr P M. Serological evidence implicating Mycoplasma genitalium in pelvic inflammatory disease. Lancet 1984; 1 : 1102-3.

13.- Cohen C R, Manhart L E, Bukusi E A, Astete S, Brunham R C, Holmes K K, et al. Association between Mycoplasma genitalium and acute endometritis. Lancet 2002; 359: 765-6.

14.- Clausen H F, Fedder J, Drasbek M, Nielsen P $\mathrm{K}$, Toft B, Ingerslev H J, et al. Serological investigation of Mycoplasma genitalium in infertile women. Hum Reprod 2001; 16 : 1866-74.

15.- Claas H C, Melchers W J, De Bruijn I H, De Graaf M, Van Dijk W C, Lindeman J, et al. Detection of Chlamydia trachomatis in 
clinical specimens by the polymerase chain reaction. Eur J Clin Microbiol Infect Dis 1990; 9: 864-8.

16.- Martínez M A, Kogan R, Rojas P, Rubilar L, Vidal R, Payá E. Diagnosis of Chlamydia pneumoniae in community acquired pneumonia in children in Chile. Acta Paediatrica 2000; 89: 650-3.

17.- Ovalle A, Martínez MA, Villagra E. Roncone R, Sandoval R, Silva R. Flora microbiana genital en embarazadas con y sin riesgo de infección. Rev Chil Obstet Ginecol 1996; 61: 5-11.

18.- Porte L, Ovalle A, Martínez M A, Santa Cruz A, Pinto M E. Comparación de la reacción de polimerasa en cadena y cultivo en la detección de Ureaplasma urealyticum en líquido amniótico. Rev Chil Infect 1999; 16: $105-11$.

19.- Balsdon M J, Martínez M A, Céspedes P, Biotti M, Saumann A, Chávez S, et al. Vaginosis bacteriana: diagnóstico mediante tinción de Gram de muestra obtenida por dos procedimientos. Rev Chil Infect 1999; 16: 100-4.

20.- Varela J A, Otero L, García M J, Palacio V, Carreño $F$, Cuesta $M$, et al. Trends in the prevalence of pathogens causing urethritis in Asturias, Spain 1989-2000. Sex Transm Dis 2003; 30: 280-3.

21.- Horner P J, Thomas B J, Gilroy C B, Egger M, Taylor-Robinson D. Role of Mycoplasma genitalium and Ureaplasma urealyticum in acute and chronic nongonococcal urethritis.
Clin Infect Dis 2001; 32: 995-1003.

22.- Thomason J L, Gelbart S M, Scaglione N J. Bacterial vaginosis: current review with indications for asymptomatic therapy. Am J Obstet Gynecol 1991; 165: 1210-6.

23.- Casin I, Vexiau-Robert D, De la Salmoniere P, Eche A, Grandry B, Janier M. High prevalence of Mycoplasma genitalium in the lower genital tract of women attending a sexually transmitted disease clinic in Paris, France. Sex Transm Dis 2002; 29: 353-9.

24.- Svenstrup H F, Nielsen P K, Drasbek M, Birkelund S, Christiansen G. Adhesion and inhibition assay of Mycoplasma genitalium and Mycoplasma pneumoniae by immunofluorescence microscopy. J Med Microbiol 2002; 51: 361-73. 